\title{
A Fifth Generation Approach to Problem-Solving in Forest Mensuration and Sampling
}

\author{
by
}

\author{
S.J. Titus ${ }^{1}$ and W.S. Adams ${ }^{2}$
}

\begin{abstract}
A new problem-solving methodology, based on fifth generation computer programming languages, is presented for use in forestry or other technically-oriented fields. It is based on the integration of exposition, data, and computer programs into a single document that is processed by the computer to produce a report. With this methodology the report, a text file, provides a central focus for the problem-solving process as it happens rather than being a reconstruction after the fact. The methodology exploits the capability of a fifth generation language, the Nested Interactive Array Language (Nial), to interpret programs and expressions written in a form close to natural language. Nial's powerful general-purpose analysis capabilities facilitate better understanding of the solution process rather than obscuring it, and the ease with which new operations can be defined in a problemoriented context allows the casual programmer access to powerful tools for solving new problems.
\end{abstract}

\section{Problem-Solving Activities}

The traditional end result of solving problems is a report that summarizes the analysis and results after the entire data collection and analysis process is completed. However, the methods and procedures used to obtain the solution are often obscured to a great extent by the summarization process. Although the use of computers is undisputed, the enormous processing power of computers is not easily brought to bear in situations that are not still in the exploratory or development phase. The problem-solver needs flexible computational tools that are as easy to work with as word processors, database managers, and graphics programs but which are free of the limitations of spreadsheets, the main user-friendly computational tool. Fifth-generation computing languages offer the opportunity to better integrate the problem-solving process and communication of the results by using a single document to link together the powerful software tools used by the problem-solver.

Problems are solved by completing a series of activities something like the following:

1. Problem description

2. Project planning

3. Data collection

4. Analysis

5. Interpretation

6. Final report preparation.
The common feature among all these activities is the exposition that describes each of them. However, the final report is often the first attempt to desribe and document the entire process. In addition, problems are often new, ill-defined, and exploratory in nature. As a consequence the solution process also evolves and develops in an unpredictable way with some or all of these steps being repeated as new information comes to light.

Three activities of the problem-solving process are of central interest here: data entry, analysis, and preparation of reports. The main focus of this paper is the integration of these activities using a powerful new computer programming language.

\section{Analysis Using a Fifth-Generation Language}

Fifth generation computer languages offer convenience, power, and the ability to use expressions that can be read in an almost natural way. The analysis process can often be conveyed with a brevity and clarity not possible with most of the traditional programming languages currently in use.

Programs in fifth-generation languages are usually written in a constrained form of natural language that uses English words to initiate computer actions that mimic common human interpretations of the words. The language used here is a general-purpose language with powerful computational capabilities as well. The language is called Nial, an acronym for "Nested Interactive Array Language". Nial was developed by Trenchard More $(1979,1981)$ and M.A. Jenkins (1985a, $1985 b)$ using the APL and Lisp languages as models. Nia retains many of the characteristics of earlier langauges which make it easy to intermix natural-language capabilities with 
conventional programming styles. The programming environment is the workspace, within which a variety of data and program objects are created and manipulated by entering expressions for evaluation by the Nial interpreter

\section{Convenient Data Definition and Organization}

Organizing data and getting it into the computer for analysis often presents a confusing obstacle for the beginner and casual programmer. Spreadsheet software, which is currently so popular, owes its success primarily to the development of convenient methods for entering tabular data common to most business and accounting activities. Problem-solving often includes analysis of data, and therefore, any methodology for doing analysis must address this obstacle. With Nial, several alternatives are possible.

One way of reducing this source of confusion is to define a data entry operation which treats incoming information as a mixture of words and numbers rather than as the Nial expressions typically entered at the keyboard. By setting out a few simple rules that restrict the data to a table or list format, it is possible to define a data translation operation that retains the simplicity of spreadsheet tables.

The following two examples show creation of a data object called "logs". The first shows the data as it would be entered at the keyboard However, since entering six lines without mistake is difficult, several alternatives are possible. The second example shows a much simpler expression which imports the data from a file created by any standard text editor and containing the same data but without the " $\&$ " symbol and quote marks.

\begin{tabular}{|c|c|c|c|}
\hline \multirow{5}{*}{ 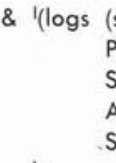 } & \multicolumn{2}{|c|}{ (species } & length diameters)) \\
\hline & PI & 4.9 & $\left(\begin{array}{ll}100 & 110\end{array}\right)$ \\
\hline & Sw & 5.2 & $\left(\begin{array}{ll}99 & 105\end{array}\right)$ \\
\hline & A & 5 & $\left(\begin{array}{ll}75 & 85\end{array}\right)$ \\
\hline & Sw & 4.9 & (109 118) \\
\hline
\end{tabular}

\& getfile "datafile;

The "\&" symbol was chosen to identify this data translation operation because it is suggestive of the Nial paragraph marker "\#" which identifies commentary material to be ignored by the interpreter. The table format is similar to that presented by spreadsheet software, but is more general since the table may be referred to as one object, a list of logs (rows), or as separate lists of species, lengths, and diameters (columns). This operation also permits single row (or column) tables to be defined. Other data structures may be defined using brackets and parentheses. For example, diameters is actually a list of both small and large end diameters for each log. The first item evaluated by the " $\&$ " operation is the parenthesized names for rows and columns of the data objects being created. Single quote marks are used to identify the data table as an array of characters rather than Nial expressions to be evaluated.

Data may also be entered directly at the keyboard. This is convenient for small data sets, but it means that the data must follow the rules Nial uses to interpret keyboard entries. This includes the requirement that non-numeric data, such as species codes, be identified as a particular type of Nial object. Common types include phrases (words beginning with a double quote), character strings (set off with single quotes), or numbers. A major reason for developing the " $\&$ " data translation operation was to avoid the necessity of flagging individual data elements, such as species codes, as phrases or character strings.

Large data sets are often maintained in separate files which may be read as character strings and converted into data objects. This procedure is more general than formatted methods provided by other languages, but requires that appropriate operations be defined to convert the characters into desired data objects. The "\&" operation is one example of this approach. A number of other operations have been developed and are available for common situations including formatting, selecting, transforming, and summarizing data as it is read.

\section{Easily Defined Problem-Oriented Vocabulary}

Although Nial supports most styles of programming and thus has a large built-in operations vocabulary, one seldom needs more than small subset to create a useful working vocabulary. Examples of these basic operations include many that have the expected everyday interpretation: sum, tally, power, In, log, exp, sqrt, abs, sin, etc. In addition, Nial allows the definition of new vocabulary relevant to the problem being solved. The following example from basic statistics illustrates how simple it is to define expressions useful for making basic data summaries. The task is to compute the average and standard deviation for a sample of log lengths recorded in metres.

$\begin{array}{cll}\text { \& }(\operatorname{logs} \text { (species } & \text { length } & \text { diameters)) } \\ \text { PI } & 4.9 & (100110) \\ \text { Sw } & 5.2 & (99105) \\ \text { A } & 5 & \left(\begin{array}{rr}75 & 85\end{array}\right) \\ \text { Sw } & 4.9 & (109118)\end{array}$

average is divide [sum, tally];

deviations is subtract [items, average];

degrees_of freedom is -1 plus tally;

variance is divide [sum (square of the deviations), degrees_of_freedom];

standard_deviation in sqrt of variance;

[average, standard_deviation] each length of logs 5. 0.1414213562

This example also illustrates the power of the array orientation taken in Nial. The basic operations (sum, tally, square) used in defining expressions like average and deviations operate on data lists or arrays and avoid the need to define looping structures to obtain sums and counts of the data. Other tools like "each", used here to extract the lengths of each log, make it possible to apply any expression to each item in a data list without having to deal with the cumbersome subroutine mechanisms of traditional languages.

\section{Script Files}

Any sequence of Nial expressions may be entered interactively at the keyboard or the sequence may be formalized in a "script" file. This is nothing more than a text file interpreted by Nial as if it had been entered at the keyboard and is similar in many ways to programs or macros common in other languages. It allows convenient integration of small data sets and analysis expressions in the same file.

The user can subdivide the script into paragraphs depending on how it is to be interpreted - as commentary (\#), as data tables (\&), or as Nial expressions to be evaluated. The 
three types of paragraphs can be intermixed at will and organized in a logical way to solve the problem at hand. Comment paragraphs and powerful operations and expressions allow the emphasis to shift from mostly program to mostly text so that the script looks more like a report. It is this ability to partition the script file that makes it possible to fully integrate the report and analysis.

The smooth linkage of Nial with the host operating environment provides powerful additional tools for analysis. Data as well as predefined operations are easily retrieved for analysis and both are stored as text files for easy editing using the standard operating system file editor. Programs written in other langages can be run to provide increased execution speed for complex tasks. Text file output from these programs can be imported into the Nial workspace for further analysis or incorporation into the report.

\section{Functional Reports}

The problem-solving process has traditionally been described by outlining the steps used in the final report. Ideally, this allows the reader of a report to duplicate the work if necessary. However, because it is summarized many technical details are obscured or left out. Data reduction and analysis programs, when written in traditional langauges, are seldom included because they are lengthy and difficult to read. Modification for other applications is risky since changes may have obscure side-effects. Languages like Nial, allow better communication of the analysis process by using fewer words with more familiar meaning to express actions taken by the computer.

Script files focus on the processing and analysis steps with less emphasis on descriptive material, while reports are strictly descriptive documents with inserted extracts of results produced by computer programs. Fifth-generation languages make it easier to integrate the script with the report so that the report file truly expresses the complete solution procedure without hiding the essential components of the analysis. The result is a functional report that consists of both exposition and operations that conduct the analysis, generate the results, and solve the problem.

A very important side-effect of using succinct functional text files is that if a particular process needs to be repeated frequently or involves large amounts of data, then the precise description of the process is available as a prototype for rewriting it in terms of machine-oriented languages like FORTRAN or $\mathrm{C}$. In the long run, such rewriting will be done by automatic compilers as required. At present, professional programmers are needed, but their task is very much simplified (and much less expensive) if they have a precise working model to follow.

\section{Example: An Inventory Report}

Point sampling (Husch, Miller, and Beers 1982) provides a familiar example that illustrates the power and clarity possible in solving real problems. The objective is to estimate basal area, number of trees, and volume per hectare for a sample of points located in a forest stand. The presentation below shows a script text file exactly as it would appear in the computer file, including commentary (\#), data (\&), and Nial expressions with the result of their evaluation following. Because a common problem-oriented vocabulary is used, the distinction between program and text is not always clear, especially to the casual observer. This feature enhances the communication process and encourages a programming style useful to both programmer and report reader. The example can be viewed as a working report that could be altered slightly by removing (\#) and data (\&) flags for inclusion in a final report.

\# Point sampling is a very common sampling technique used to estimate the average volume per hectare of standing trees in an area. The sample consists of a number of systematic points located throughout the forest. At each sample point tree data are measured if the tree is within a distance calculated as the plot radius factor, a function of the basal area factor, times the diameter of the tree. The data are organized as a list of point samples grouped here using square brackets ([]). Each point sample consists of a plot number followed by a list of trees for which species, diameter $(\mathrm{cm})$, height $(\mathrm{m})$ have been recorded:

\& '(pointsamples)

[1201, Aw $12.5 \quad 25.0$ PI 25.126 .5 Sw 50.5 35.0]

[1202, Aw 10.1 12.0 Sw $22.0 \quad 24.0$

[1203, Aw 22.0 20.0, Aw $18.2 \quad 16.9$ Aw $14.0 \quad 15.5$ Sw 24.126 .5

I; tally pointsamples 3

\# For a pointsample: pointnumber is first;

trees is rest:

each (tally trees) of pointsamples

$\begin{array}{llll}3 & 2 & 4\end{array}$

\# For this example, each sample tree represents 4 square metres per hectare of basal area. This value is constant regardless of tree size and is called the basal area factor (baf). Additional expressions are defined to estimate tree volume in cubic metres and compute the number of trees per hectare represented by each sample tree, the tree expansion factor (trf). Arbitrary coefficients are used for the volume function.

\# For a tree:

spp is first;

$\mathrm{dbh}$ is second;

ht is third

baf is 4 ;

ba is pi ${ }^{*}$ square $\left(1 / 200^{*} \mathrm{dbh}\right)$;

trf is Baf / ba;

volume is operation tree begin coeffs gets ("SW .0000455 $1.816 \quad 1.074$ ) ("PL .0000327 1.9221 .113 ) ("AW . 0000245 $2.022 \quad 1.050$ );

spp gets first tree;

$\mathrm{dbh}$ gets second tree;

ht gets third tree;

there sppindex gets spp seek each first coeffs; a b c gets if there then rest (sppindex pick coeffs) else rest first coeffs endif: $a^{*}($ dbh power b) * (ht power c) end:

\# In this definition, all objects created within the operation are local and exist only while the function is being executed. The only input is a tree and the only result is the predicted tree volume. Computationally this definition is inefficient since each time the operation is called, four objects are created; on the other hand, the operation is totally self-contained and depends only on the tree input data.

\# Compute values for the tree expansion factor and tree volume for the second tree of the first point sample, and show answers with three digits to the right of the decimal.

setdigits 3 ; 
[items, trf, volume] of second trees of first pointsamples

\begin{tabular}{|ll|l|l|}
\hline PL 25.1 & 26.5 & 80.839 & 0.615 \\
\hline
\end{tabular}

\# For each point sample, volume per hectare is estimated as the sum, for all trees sampled, of the tree volume times the tree expansion factor. The average volume per hectare is the avearge of volumes per hectare for each point, assuming that the points are a simple random sample. For each point sample several expressions define the estimates of interest: basalarea__per_ha is baf times tally of trees;

trees_per_ha is sum each trf of trees;

volume__per_ha is sum each (product [volume, trf]) of trees;

estimates is [volume__ per_ha, trees__per_ha, basalarea_per_ha]; average is divide [sum, tally];

\# With these expressions it is easy to estimate volume, number of trees, and basal area per hectare for any or all point samples:

estimates of second pointsamples

57.679604 .4878

stack each estimates of pointsamples

\begin{tabular}{|c|}
\hline $\begin{array}{lll}139.717 & 426.759 & 12\end{array}$ \\
\hline $57.679 \quad 604.4878$ \\
\hline $122.556 \quad 606.513 \quad 16$ \\
\hline
\end{tabular}

average each estimates of pointsamples

106.651545 .9212$.

\section{Conclusions}

The ease of dealing with text documents in a computer system and the ability to complete complicated data analysis with very high level language systems allows the test document to become the focal point of problem solving at the beginning of the process rather than the end. The initial document can be a first draft that evolves into the final report as the various activities are completed. The actual data analysis can be included in this process because of the power and convenience of these new langauges.

The methodology outlined here allows the forester or any other worker in a technically-oriented field to use computers without major digressions from the standard practices and terminology used in his field. The text file serves as the medium for acquiring data, manipulating it and reporting results. The file can be stored in an archive or communicated to others without special techniques being invoked. It can also be processed by a variety of independent programs (or people) because the only assumption made is that it is encoded in standard text form.

Because programs written in fifth-generation languages are compact and often can be read as if they were ordinary natural language expositions, it is feasible to incorporate the complete description of dats processing operations directly in technical reports. Reports destined for upper levels of any agency carry a reassuring measure of authority because of their completeness. Similarly, such reports give people who generate the data a better understanding of the overall purpose of their work.

\section{References}

Jenkins, M.A. and W.H. Jenkins, 1985a. Q'Nial Reference Manual, Brown and Martin Ltd, Kingston, Ontario. $461 \mathrm{pp}$.

Jenkins, M.A. 1985b. Q'Nial Tutorial. Brown and Martin Ltd, Kingston, Ontario. 183 pp.

Husch, B.H., C.I. Miller, and T.W. Beers. 1982. Forest Mensuration. John Wiley \& Sons, New York. 402 pp.

More, T. 1979. The Nested Rectangular Array as a Model of Data. In Proc. ACM - APL79 Conf., 30 May - 1 June 1979 Rochester, N.Y. APL Quote Quad Vol 9 (4 Part 1): 55-74.

More, T. 1981. Notes on the Diagrams, Logic, and Operations of Array Theory. IBM Technical Report GC320-2137. IBM Scientific Center, Cambridge. 170 pp. 